ARTICLE

\title{
A physicochemical orthophosphate cycle via a kinetically stable thermodynamically activated intermediate enables mild prebiotic phosphorylations
}

Oliver R. Maguire (D) ${ }^{1}$, Iris B. A. Smokers (iD ${ }^{1} \&$ Wilhelm T. S. Huck (iD ${ }^{1 凶}$

The incorporation of orthophosphate from scarce geochemical sources into the organic compounds essential for life under mild conditions is a fundamental challenge for prebiotic chemistry. Here we report a prebiotic system capable of overcoming this challenge by taking inspiration from extant life's recycling of orthophosphate via its conversion into kinetically stable thermodynamically activated (KSTA) nucleotide triphosphates (e.g. ATP). We separate the activation of orthophosphate from its transfer to organic compounds by, crucially, first accumulating a KSTA phosphoramidate. We use cyanate to activate orthophosphate in aqueous solution under mild conditions and then react it with imidazole to accumulate the KSTA imidazole phosphate. In a paste, imidazole phosphate phosphorylates all the essential building blocks of life. Integration of this chemistry into a wet/dry cycle enables the continuous recycling of orthophosphate and the accretion of phosphorylated compounds. This system functions even at low reagent concentrations due to solutes concentrating during evaporation. Our system demonstrates a general strategy for how to maximise the usage of scarce resources based upon cycles which accumulate and then release activated intermediates.

\footnotetext{
${ }^{1}$ Institute for Molecules and Materials, Radboud University Nijmegen, 6525 AJ Nijmegen, The Netherlands. $凶_{\text {email: w.huck@science.ru.nl }}$
} 
$\mathrm{P}$ hosphorylated molecules play a series of diverse and essential roles in extant biology, including driving endergonic reactions via the release of free energy upon phosphate transfer, preserving genetic information due to the hydrolytic stability of phosphate diesters in the backbone of DNA and retaining cellular contents by the presence of a charged phosphate head group on both the phospholipids in the cell membrane and on compounds in the cytoplasm ${ }^{1,2}$. Given the centrality of phosphate chemistry to life, there has been widespread interest in establishing plausible pathways for the formation of prebiotically important phosphorylated organic molecules ${ }^{3-5}$.

On the early Earth, geochemical sources of orthophosphate were scarce and were most likely found in minerals such as apatite, vivianite, berlinite and struvite, or from the hydrolysis of phosphorus-containing minerals such as schreibersite ${ }^{3}$. In order for orthophosphate to be available for transfer to organic compounds, it will require its release from a mineral, which will most probably arise from dissolution of the mineral into solution. It is notable that in aqueous solution, the most thermodynamically stable form of phosphorus is orthophosphate ${ }^{6}$. Transfer of orthophosphate from these geochemical sources to prebiotically important organic compounds is therefore a key requirement for the origins of life. However, previous examples of prebiotic phosphorylation reactions, which use orthophosphate directly, require aggressive heating conditions in conjunction with an activating reagent to overcome the high kinetic barrier for the activation of orthophosphate $\mathrm{e}^{7-14}$ and/or the use of non-aqueous eutectic solvents such as urea and formamide ${ }^{15,16}$. In particular, aggressive heating conditions (typically at $100^{\circ} \mathrm{C}$ ) can have deleterious effects on other molecules through either hydrolysis or other unwanted side reactions and are likely incompatible with any form of protocellular chemistry. Alternative approaches to prebiotic phosphorylation have exploited activated sources of phosphate. Phosphoramidates, including diamidophosphate (DAP), have been highlighted as powerful prebiotic phosphorylation reagents ${ }^{17-23}$. However, a prebiotically relevant method to directly recycle orthophosphate back into DAP has not been demonstrated. Finally, given the challenges of using orthophosphate, alternative sources of phosphorus, e.g., phosphite ${ }^{6,24}$ and cyclopolyphosphates $^{25}$, have also been explored.

Considering the challenges posed by activating orthophosphate and transferring it in a single reaction step, we were inspired by extant life's biochemistry, which is reliant upon the ability of cells to continuously recycle orthophosphate back into an activated form. This cycling of orthophosphate is mediated by nucleotide triphosphates (NTPs), e.g. ATP, which preserve a kinetically stable thermodynamically activated (KSTA) form of phosphate ${ }^{26-29}$.

Here we activate orthophosphate with cyanate in aqueous solution to form carbamoyl phosphate under mild prebiotically plausible conditions. We demonstrate that this activated phosphate can be captured with imidazole to form imidazole phosphate, a KSTA molecule. Imidazole phosphate can accumulate in solution, as it is resistant to both hydrolysis and reactions with most other nucleophiles. We show that in a paste, imidazole phosphate can transfer the phosphate group to a wide range of prebiotically important organic compounds. We then adopt a systems chemistry approach ${ }^{30,31}$ whereby we demonstrate a prebiotically plausible physicochemical orthophosphate cycle by integrating this chemistry into a wet/dry cycle. The cycle is 'driven' by repeatedly 'refuelling' with fresh cyanate solution and each pass through the cycle results in a stepwise increase in phosphorylated compounds. Finally, we show how the process of solutes being concentrated as a solution evaporates in the wet/dry cycle allows us to exploit significantly lower concentrations
(10-20 mM) of cyanate, imidazole, orthophosphate and a nucleophile, and still obtain phosphorylated compounds. Our results illustrate a general strategy for how to construct a system, which maximizes the usage of scarce resources by capturing, storing and accumulating activated versions of these compounds in a KSTA molecule whose chemical energy can be later channelled towards useful functions.

\section{Results}

The formation and accumulation of imidazole phosphate in mild conditions. We reasoned that we could activate orthophosphate by converting it into carbamoyl phosphate through a reaction with isocyanate ${ }^{32,33}$ and then capture the activated phosphate with imidazole to form imidazole phosphate (Fig. 1a). In a solution containing $450 \mathrm{mM}$ potassium cyanate, $150 \mathrm{mM}$ sodium phosphate monobasic and 1.0 M imidazole at $\mathrm{pH} 7.32$, we observed by ${ }^{31} \mathrm{P}$ nuclear magnetic resonance (NMR) spectroscopy the accumulation of imidazole phosphate in a yield of $25 \%$ after 4 days at $22^{\circ} \mathrm{C}$ (Fig. 1b, c). Initially, carbamoyl phosphate was formed and this was converted into imidazole phosphate via substitution reaction with imidazole (Fig. 1b, c). Separate experiments confirmed that imidazole phosphate was formed directly from carbamoyl phosphate (Supplementary Information (SI) Section S2.3). Excess cyanate concentration relative to orthophosphate was added, in order to push the equilibrium toward the formation of carbamoyl phosphate ${ }^{32,33}$. After 3 days, the concentration of imidazole phosphate decreased slowly over time due to hydrolysis (Fig. 1c). Imidazole phosphate was formed in yields of between $18 \%$ and $25 \%$ across $\mathrm{pH} 6.37-7.32$ and the addition of metal ions $\mathrm{Mg}^{2+}$ and $\mathrm{Zn}^{2+}$ (typically used as Lewis acid catalysts in prebiotic chemistry) did not significantly affect the yield of imidazole phosphate formed (Fig. 1d and SI Sections S2.2 and S2.3). Under identical conditions, other substituted imidazoles and pyrazole also formed their respective imidazole phosphates, albeit in a lower yield than imidazole (5-20\%), thereby demonstrating the generality of this approach for capturing activated phosphate (SI Section S2.4).

Imidazole phosphate is a good candidate for a prebiotically plausible KSTA molecule. Imidazole phosphate has reasonable kinetic stability towards hydrolysis with a half-life of $t_{1 / 2}=23.1 \mathrm{~h}$ at $\mathrm{pH} 7.0$ and $39^{\circ} \mathrm{C}^{34,35}$. Whether imidazole phosphate can be regarded as thermodynamically activated depends upon whether phosphate transfer from imidazole phosphate to a nucleophile is thermodynamically favoured. An initial assessment of this can be done by comparing the Gibbs free energies of hydrolysis $\left(\Delta G_{\text {hyd }}\right)$ of imidazole phosphate with that of the phosphorylated nucleophile. The hydrolysis of imidazole phosphate has a $\Delta G_{\text {hyd }}\left[\mathrm{pH} \quad 7.4,25^{\circ} \mathrm{C}\right]=-32.0 \mathrm{~kJ} \mathrm{~mol}^{-136}$. Thus, if $\Delta G_{\mathrm{hyd}}>$ $-32.0 \mathrm{~kJ} \mathrm{~mol}^{-1}$ for the phosphorylated nucleophile, then phosphate transfer is favourable and the imidazole phosphate can be described as thermodynamically activated. For example, glycerol1-phosphate has $\Delta G_{\text {hyd }}=-9.2 \mathrm{~kJ} \mathrm{~mol}^{-1}$ and, therefore, imidazole phosphate is thermodynamically activated relative to glycerol ${ }^{37}$. Various prebiotic syntheses of imidazole are known 38,39 .

Phosphate transfer to the essential building blocks of life in a paste. Next, we set out to confirm whether imidazole phosphate would be kinetically stable towards a wide range of prebiotically relevant organic compounds in aqueous solution. Amino acids, nucleosides, nucleotides and sugars were added to solutions containing synthetically prepared calcium/sodium imidazole phosphate ${ }^{40}$ (SI Section S3) in citric acid buffered solutions at $\mathrm{pH}$ 3.0-6.9 and $22^{\circ} \mathrm{C}$ (see SI Section S4.1 and Supplementary Table 1). In all bar two cases with hydroxylamines (see SI Section S4), no transfer of phosphate to these nucleophiles was observed 


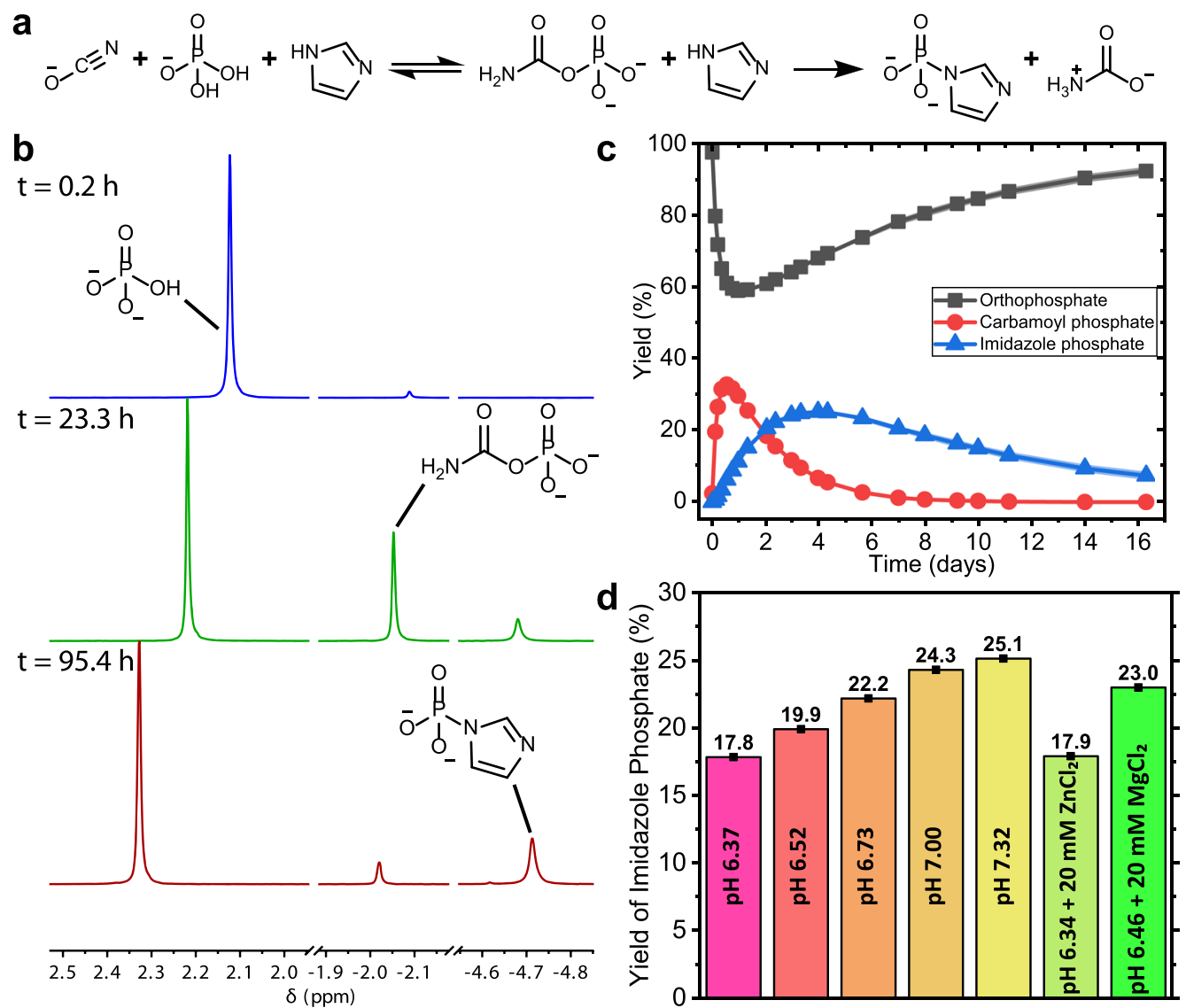

Fig. 1 The formation of imidazole phosphate in mild prebiotic conditions. a The reaction steps for the formation of imidazole phosphate from orthophosphate, cyanate and imidazole. Cyanate isomerizes to isocyanate prior to reacting with orthophosphate to form carbamoyl phosphate. b Representative ${ }^{31}$ P-NMR spectra over time for the formation of imidazole phosphate from $450 \mathrm{mM}$ potassium cyanate $+150 \mathrm{mM}$ sodium phosphate in $1.0 \mathrm{M}$ imidazole buffer at $22^{\circ} \mathrm{C}$ and $\mathrm{pH}$ 7.32. A minor increase in $\mathrm{pH}$ over the course of the reaction causes the change in chemical shift of peaks. $\mathbf{c}$ Profile of the changing yield of phosphorus-containing species over time for the reaction shown in $\mathbf{b}$. Shaded area around traces is the SD from triplicate experiments. $\mathbf{d}$ The effect of $\mathrm{pH}$ and metal ions on the yield of imidazole phosphate. All reactions in $\mathbf{d}$ used identical concentrations as detailed in $\mathbf{b}$.

in solution and only a slow hydrolysis of imidazole phosphate took place. Overall, this confirmed that in solution, imidazole phosphate is kinetically stable towards almost all organic nucleophiles as well as to hydrolysis. Thus, activated phosphate could be accumulated in solution in the form of imidazole phosphate.

Prebiotic catalysts for phosphate transfer reactions in solution that are analogous to the role of kinases in extant life are currently unknown, but we reasoned that by switching to paste conditions, where the activity of water is decreased, we would be able to transfer the phosphate from imidazole phosphate to oxygen-based nucleophiles apart from water ${ }^{3,20}$. We conducted a series of paste reactions with a diverse set of prebiotically important organic molecules: amphiphile precursors ${ }^{41}$, amino acids/peptides ${ }^{42-44}$, metabolites $^{45,46}$, nucleosides ${ }^{47,48}$, nucleotides and sugars ${ }^{49}$. We made the pastes either by adding a small amount of water to the solid compounds or by dissolving the solid compounds in water and then waiting for the solution to evaporate into a paste via a wet-to-dry transition. In the first set of paste reactions, we mixed solid calcium imidazole phosphate with solid/liquid nucleophile and added water $\left(0.1-0.2 \mu \mathrm{L} \mathrm{mg}^{-1}\right)$ to form a paste and the pastes were heated to $50^{\circ} \mathrm{C}$ or $80^{\circ} \mathrm{C}$ for between 24 and $168 \mathrm{~h}$ (1-7 days) upon which phosphate transfer was observed in yields of up to $52 \%$ (SI Sections S5 and S6.8, and Supplementary Table 2). Yields at $50{ }^{\circ} \mathrm{C}$ were typically better than at $80^{\circ} \mathrm{C}$. In the second method, we demonstrated that the phosphate transfer reactions could occur after a wet-to-dry transition (Table 1, Fig. 2, and SI Section
S6). We dissolved calcium imidazole phosphate and the nucleophile in water, and left the solution to dry at $22^{\circ} \mathrm{C}$ for 12-48 h. Notably, in several cases, the addition of the nucleophile to the solution aided the dissolution of calcium imidazole phosphate, presumably by coordinating to the $\mathrm{Ca}^{2+}$ ion. The presence of organic compounds in solution has, along with cyanide ions, previously been shown to help solubilize calcium phosphate minerals such as apatite ${ }^{9,50}$. The resultant paste was then heated to $50^{\circ} \mathrm{C}$, to promote phosphate transfer. The reactions were followed by the periodic removal of a sample from the paste and analysed using ${ }^{31} \mathrm{P}-\mathrm{NMR}$ spectroscopy. The transfer of the phosphate group from imidazole phosphate in the paste was relatively facile with most reactions going to completion after 24-72 h. In the paste, two other reactions were observed: the hydrolysis of imidazole phosphate and the formation of pyrophosphate $^{51}$ via the reaction of imidazole phosphate with either itself or with orthophosphate. To counter the formation of pyrophosphate, we used a five-fold excess of the nucleophile. Spiking experiments with genuine samples of phosphorylated compounds confirmed the identity of phosphorylated compounds in the ${ }^{31} \mathrm{P}-\mathrm{NMR}$ spectra (SI Section S6).

A wide array of prebiotically important organic compounds were phosphorylated using imidazole phosphate in a paste reaction (Table 1). The amphiphile precursor glycerol phosphate, a key component for the formation of phospholipids with the two hydrophobic tails essential for bilayer formation ${ }^{41}$, was formed in a yield of $38 \%$, with glycerol-1-phosphate and glycerol-2- 
Table 1 The amphiphile precursors, amino acids + peptides, metabolites, nucleosides, nucleotides and sugars phosphorylated by imidazole phosphate under paste conditions (see SI Section S6).

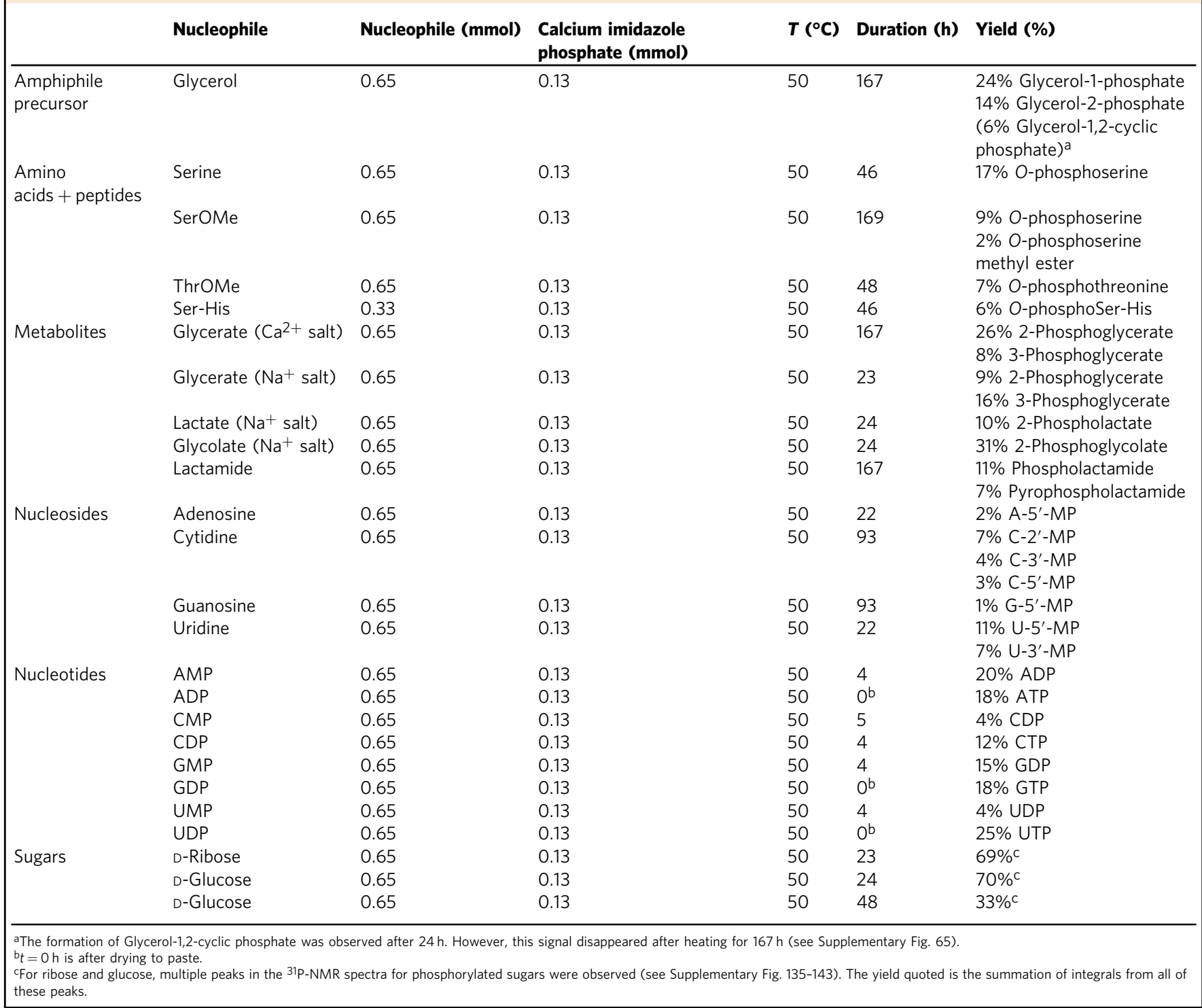

phosphate formed in a $24 \%$ and $14 \%$ yield, respectively (Fig. 2). The hydroxyl side groups of amino acids and amino acid residues in peptides, such as serine, were phosphorylated in $6-17 \%$ yield. Organic compounds that in extant life function as metabolites and in prebiotic chemistry can act as phosphorylated building blocks were formed in a $10-34 \%$ yield. A precursor for phosphoenolpyruvate (biology's highest-energy phosphate), 2phosphoglycerate, was formed in a $9 \%$ yield. Nucleosides were converted into nucleotide monophosphates in up to an $18 \%$ yield. Sugars were phosphorylated in yields of 33-70\% including ribose, an important precursor for ribonucleotides, and glucose. The phosphorylation of the sugars was not regioselective and the yields quoted in Table 1 reflect the combined yield of all phosphorylated sugar products. Spiking experiments confirmed the formation of ribose-1-phosphate, ribose-5-phosphate, glucose-1-phosphate and glucose-6-phosphate, but other phosphorylated sugars were also observed. In addition to phosphate monoesters, imidazole phosphate could also synthesize oligophosphates via the formation of a phosphate anhydride bond. Nucleotide-5'-monophosphates (NMPs) were converted into nucleotide diphosphates (NDPs) in a $4-20 \%$ yield and NDPs could be converted to NTPs in yields of between $3 \%$ and $25 \%$, with the two NTPs predominantly used in cellular biochemistry, ATP and GTP, both being formed in an 18\% yield. Control experiments performed in the absence of imidazole phosphate confirmed that the NDPs were formed exclusively from the reaction of the NMP with imidazole phosphate as opposed to phosphate transfer between two NMPs and control reactions with NDPs confirmed that either all or the majority of NTPs were formed from the reaction with imidazole phosphate (SI Section S6.6). The formation of NDPs and NTPs is particularly encouraging, as it provides a direct link between this prebiotic chemistry and the chemistry that life eventually settled on for the cycling of orthophosphate.

A physicochemical orthophosphate cycle for prebiotic phosphorylations. Prebiotic sources of free orthophosphate are likely to be limiting ${ }^{3}$ and thus demonstrating a prebiotically plausible route to recycle orthophosphate is crucial. We reasoned that we could construct such an orthophosphate cycle by integrating our above results together into a wet/dry cycle (Fig. 3a). In solution, orthophosphate can be activated via a reaction with cyanate and 


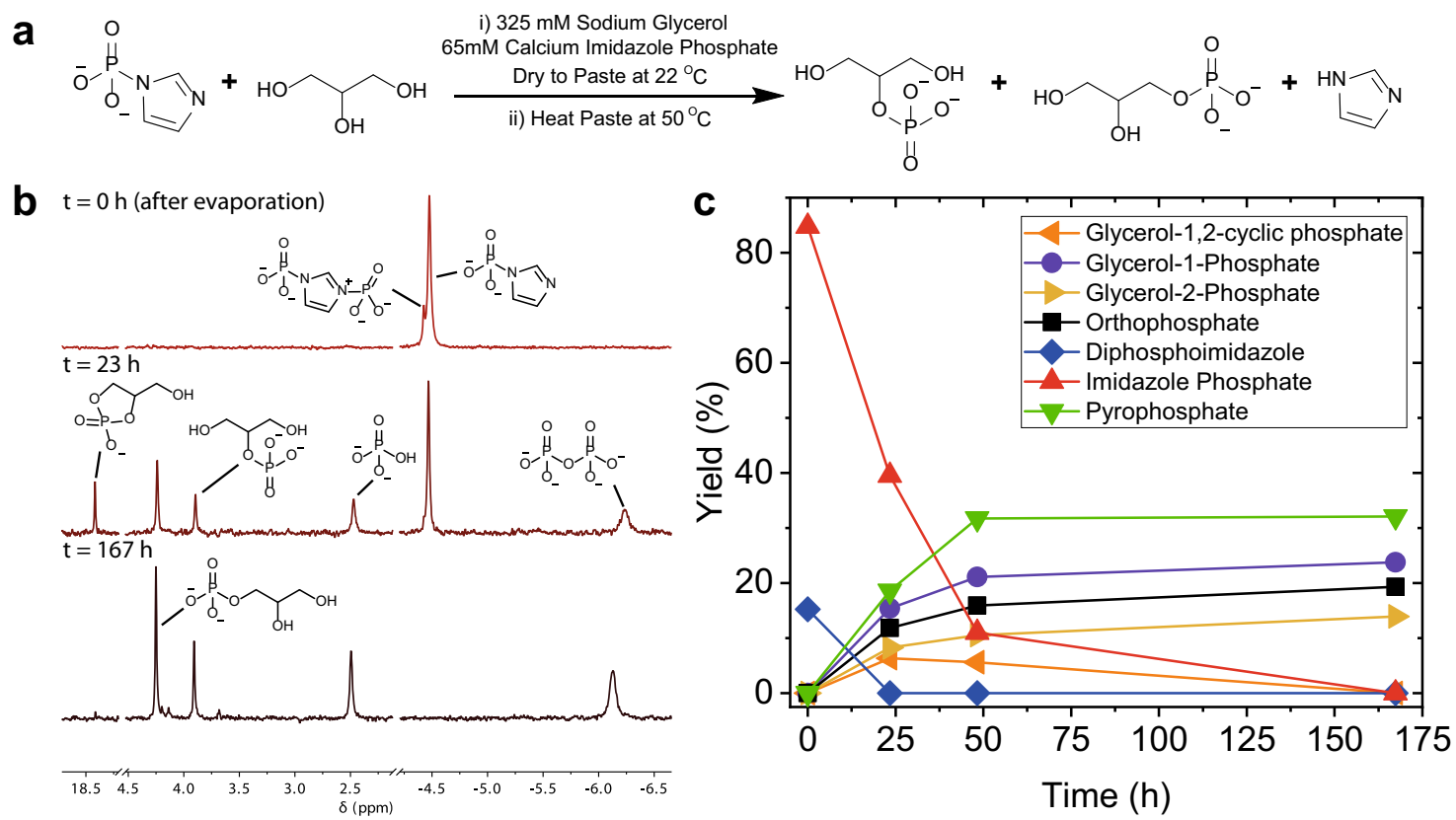

Fig. 2 The phosphorylation of glycerol by imidazole phosphate in paste conditions. a The phosphorylation reaction of glycerol by calcium imidazole phosphate in paste conditions after drying to a paste using the second method. b Representative ${ }^{31} \mathrm{P}-\mathrm{NMR}$ spectra over time for the reaction of $0.13 \mathrm{mmol}$ calcium imidazole phosphate and $0.65 \mathrm{mmol}$ glycerol in paste conditions at $50^{\circ} \mathrm{C}$. The paste was prepared by leaving $2.0 \mathrm{~mL}$ of a $65 \mathrm{mM}$ calcium imidazole phosphate and $325 \mathrm{mM}$ glycerol solution to dry for $48 \mathrm{~h}$ at $22^{\circ} \mathrm{C}$. The ${ }^{31} \mathrm{P}$-NMR spectrum at $t=0 \mathrm{~h}$ was taken from the paste after the evaporation of water. During the drying stage diphosphoimidazole formed from the reaction of imidazole phosphate with itself. $\mathbf{c}$ Yields of phosphatecontaining compounds over time for the reaction in $\mathbf{a}$ and $\mathbf{b}$.

converted into imidazole phosphate. Upon drying to a paste and heating, the phosphate can be transferred from imidazole phosphate to prebiotically important organic compounds. The cycle can then be completed by redissolving the paste in a cyanate solution, thereby 'refuelling' the system to reactivate the remaining orthophosphate and regenerate imidazole phosphate. Repeated cycling would lead to the accumulation of phosphorylated compounds.

To demonstrate the orthophosphate cycling, we first prepared a $450 \mathrm{mM}$ potassium cyanate, $1.0 \mathrm{M}$ imidazole and $20 \mathrm{mM}$ sodium phosphate solution with $750 \mathrm{mM}$ of a nucleophile (glycerol, glycerate, serine, AMP or the nucleoside cytidine) (SI Section S7.1-S7.4 and S7.9). The $\mathrm{pH}$ of the solution was adjusted to $\mathrm{pH} 7.3$ and left to react at $22^{\circ} \mathrm{C}$ for $24 \mathrm{~h}$, in order to accumulate imidazole phosphate. The solution was then dried to a paste at $22{ }^{\circ} \mathrm{C}$ over a period of $24 \mathrm{~h}$. The resultant paste contained both imidazole phosphate and phosphorylated nucleophiles, thereby demonstrating that phosphate transfer from imidazole phosphate to the nucleophile occurred during the drying process. The paste was then heated to $50{ }^{\circ} \mathrm{C}$ for $24 \mathrm{~h}$, to promote further phosphate transfer to the nucleophile. Pyrophosphate and triphosphate were formed as side products and hydrolysis of imidazole phosphate back to orthophosphate occurred via a reaction with residual water ${ }^{51}$. The cycle was completed by redissolving the paste in $450 \mathrm{mM}$ potassium cyanate solution, in order to reactivate orthophosphate and accumulate imidazole phosphate once again. Three repetitions of the cycle were performed and the percentage of orthophosphate incorporated into the organic compounds at the end of each cycle (referred to as 'Yield' on the $y$-axis) are shown in Fig. 3b-e. A stepwise increase in phosphorylated compounds was observed predominantly between the first and second cycle for all the nucleophiles. Further cycling experiments with other nucleophiles including serine, D-ribose, D-glucose and the nucleoside adenosine showed similar trends (SI Section S7.5-S7.8). When the physicochemical cycle was performed with higher concentrations of orthophosphate of $50 \mathrm{mM}$ and $150 \mathrm{mM}$, similar trends were also observed (see SI Section S7.2-S7.9). Previous reports have shown phosphate anhydride bond and phosphate monoester formation in cyanate and orthophosphate solutions, albeit usually in very poor yields ${ }^{52-55}$. To ascertain whether this was occurring in our experiments, we performed control reactions in the absence of imidazole (SI Section S7.10). These showed no formation of phosphorylated compounds (or pyrophosphate or triphosphate), thereby confirming that the reagent for phosphate transfer in the pastes was imidazole phosphate and not carbamoyl phosphate. We assume that differences in the conditions between our control experiments and previous studies account for the absence of phosphorylation under these circumstances.

The concentrations of potassium cyanate, imidazole and the nucleophile used in the above experiments were high. This was done in order to clearly observe experimentally the formation and accumulation of imidazole phosphate and identify how the reactions in the cycle proceeded. Nevertheless, such high concentrations are less prebiotically plausible. However, the concentration of any solution is relative to the volume of its solvent and we therefore reasoned that we could use the wet/dry cycle to provide a prebiotically plausible means to access higher concentrations. As solvent water evaporates, the concentration of solutes in solution increases. We therefore chose to perform the physicochemical orthophosphate cycling with concentrations of potassium cyanate, imidazole and a nucleophile at $100 \mathrm{mM}$ level with a sodium phosphate concentration of $20 \mathrm{mM}$ (Fig. 3f-i) and $50 \mathrm{mM}$ (SI Section S7.11). An $\sim 100 \mathrm{mM}$ concentration range is typical of that used in prebiotic chemistry $44,46,56$. Here, the stepwise increase in the incorporation of orthophosphate into phosphorylated nucleophiles still functions. For the different nucleophiles, the percentage of orthophosphate incorporated into the phosphorylated nucleophile after three cycles is on average 


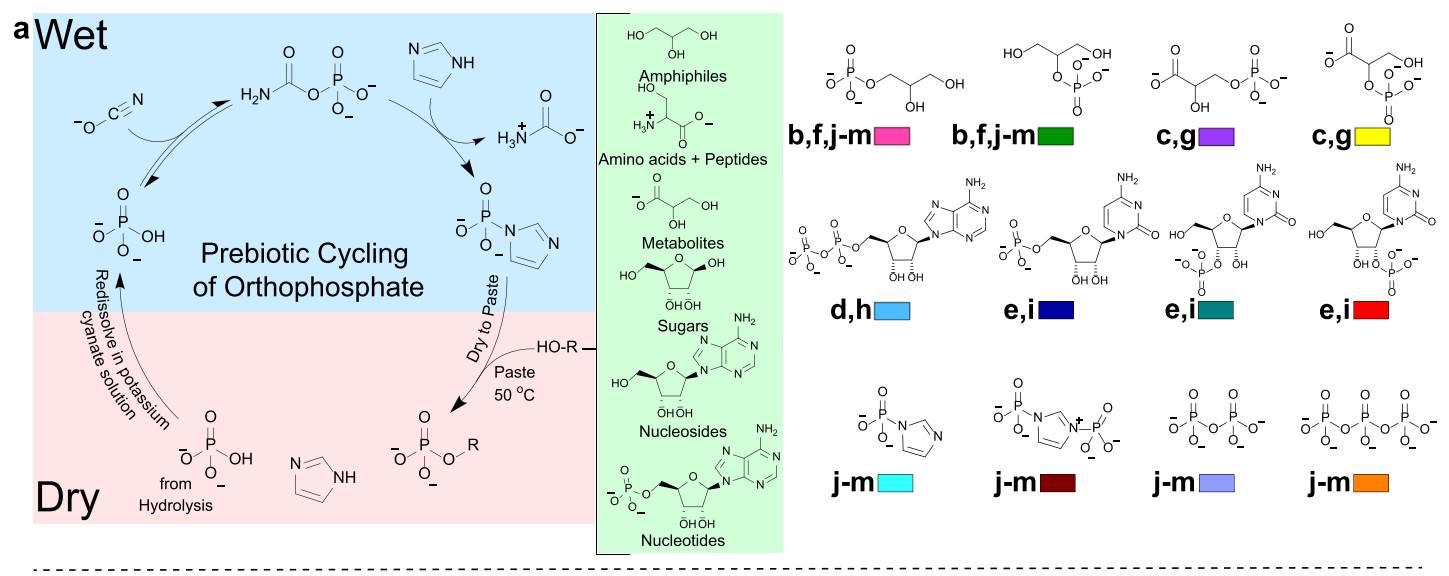

$[\mathrm{KOCN}]=450 \mathrm{mM},\left[\mathrm{Na}_{2} \mathrm{HPO}_{4}\right]=20 \mathrm{mM},[$ Imidazole $]=1000 \mathrm{mM},[$ Nucleophile $]=750 \mathrm{mM}$
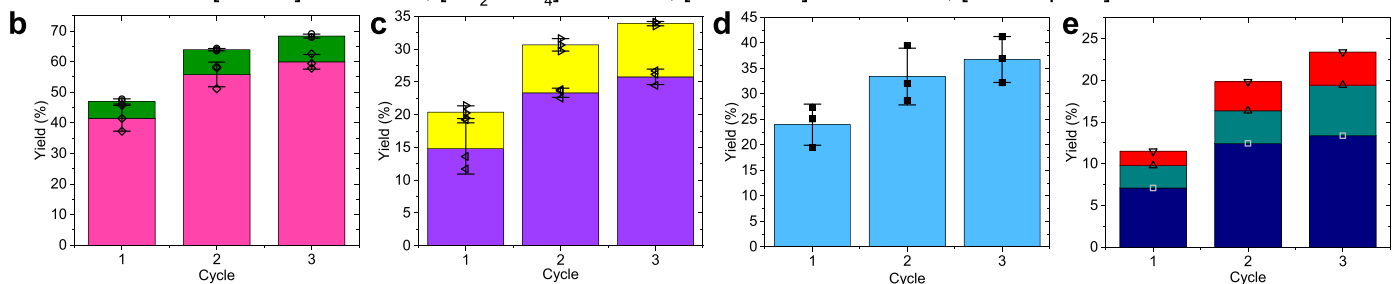

$[\mathrm{KOCN}]=100 \mathrm{mM},\left[\mathrm{Na}_{2} \mathrm{HPO}_{4}\right]=20 \mathrm{mM},[$ Imidazole $]=100 \mathrm{mM},[$ Nucleophile $]=100 \mathrm{mM}$
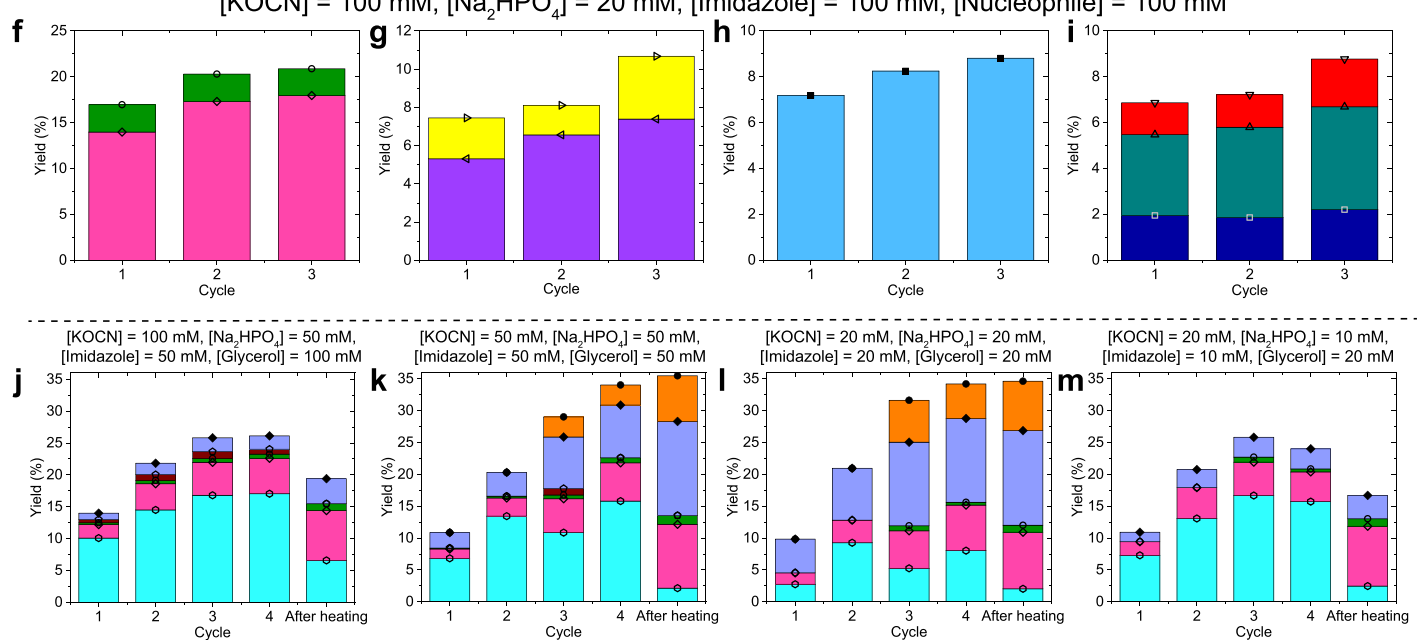

Fig. 3 The physicochemical orthophosphate cycle, which upon repeated cycling enables a stepwise increase in the incorporation of orthophosphate into prebiotically important organic compounds. a Overview of the orthophosphate cycling experiment. The second and third cycles were performed by redissolving the paste in a solution with the respective potassium cyanate concentration for each set of experiments. The plots $\mathbf{b}$ - $\mathbf{m}$ show the yield of incorporation of orthophosphate into phosphorylated products over the course of three cycles and this is referred to as 'Yield' on the $y$-axis. b-e Experiments initiated from a solution of $450 \mathrm{mM}$ potassium cyanate, $1.0 \mathrm{M}$ imidazole and $20 \mathrm{mM}$ sodium phosphate with $750 \mathrm{mM}$ of the nucleophile (glycerol, glycerate, AMP and the nucleoside cytidine). Error bars are based on the SD determined from three repeats. $\mathbf{f}-\mathbf{i}$ Experiments initiated from a solution of $100 \mathrm{mM}$ potassium cyanate, $100 \mathrm{M}$ imidazole and $20 \mathrm{mM}$ sodium phosphate with $100 \mathrm{mM}$ of the nucleophile (glycerol, glycerate, AMP and the nucleoside cytidine). $\mathbf{j}-\mathbf{m}$ Wet/dry cycling experiments performed at lower initial concentrations in the presence of glycerol, which demonstrate the formation of imidazole phosphate as the solution concentrates while drying and the transfer of phosphate to the nucleophile in the paste. To emphasize the process of a solution concentrating as it dries, the yield of imidazole phosphate and all products that it forms are shown.

only 3.2-fold lower than those for the higher concentrations above, despite the concentrations of cyanate, imidazole and nucleophile being lowered by between 4.5 - and 10 -fold. The cycle was also performed with serine as the nucleophile (SI Section S7.11.8 and S7.11.9).

Finally, we decided to probe whether this physicochemical orthophosphate cycle would still function well at much lower initial concentrations of potassium cyanate, orthophosphate, imidazole and the nucleophile glycerol. We prepared solutions with concentrations reduced by up to 100 -fold compared to those above, with imidazole and sodium phosphate concentrations of
10-50 $\mathrm{mM}$, and potassium cyanate and glycerol concentrations of 20-100 mM (Fig. 3j-m and SI Section S7.12). These solutions were left for $\sim 24 \mathrm{~h}$ before being dried to a paste. To demonstrate that imidazole phosphate can accumulate over the course of several cycles even at these much lower concentrations, we applied heating to the pastes $\left(50^{\circ} \mathrm{C}\right.$ for $\left.31 \mathrm{~h}\right)$ only after four cycles. Fig. 3j-m demonstrates that even at these low initial concentrations, the effect of a solution concentrating as it evaporates enabled the formation of imidazole phosphate and the transfer of the phosphate in the paste to the glycerol. For example, Fig. $3 \mathrm{~m}$, where reagents were in the $10-20 \mathrm{mM}$ range, 
shows that $16 \%$ of the orthophosphate was accumulated into imidazole phosphate after four cycles, thereby demonstrating its ability to function as a KSTA molecule. In Fig. $3 \mathrm{~m}, 5 \%$ of the orthophosphate was incorporated into phosphorylated glycerol over the four cycles, which was elevated to $11 \%$ after heating. In Fig. $3 j-m$, the yield of incorporation of the orthophosphate into phosphorylated glycerol at these lower concentrations was reduced compared to those experiments at the higher concentrations above, but we do not believe this to be a significant issue, as these wet/dry cycles could have occurred multiples times on the early Earth and the resulting phosphate monoester products generally have good hydrolytic stability, which will enable their accretion. Fig. $3 \mathrm{j}-\mathrm{m}$ also include the side products diphosphoimidazole, pyrophosphate and triphosphate (which are all formed via imidazole phosphate) in order to demonstrate that even at these lower concentrations, overall between $26 \%$ and $35 \%$ of the orthophosphate was converted into imidazole phosphate over the course of four cycles. For Fig. 3j, m, after the heating was applied, the overall percentage incorporation of orthophosphate dropped due to hydrolysis of imidazole phosphate. The formation of these aforementioned side products was more significant when all reagents were present in an equivalent concentration (Fig. 3k, l) than when the concentrations of cyanate and glycerol were twice that of orthophosphate and imidazole (Fig. 3j, m). We therefore demonstrate here that even at lower initial concentrations of all reagents, this physicochemical orthophosphate cycle still functions well.

\section{Discussion}

Here we have demonstrated a prebiotically plausible system by which orthophosphate can be activated, accumulated in a KSTA compound in solution, transferred to prebiotically important organic compounds in a paste and repeatedly recycled all within a wet/dry cycle (Fig. 3a). Orthophosphate was activated under mild conditions using cyanate to form carbamoyl phosphate and this activated phosphate was captured and stored via the formation of the phosphoramidate imidazole phosphate. The imidazole phosphate is kinetically stable towards hydrolysis and thus an activated form of phosphate can accumulate in solution as a KSTA molecule. Phosphate transfer from imidazole phosphate to all classes of the essential organic molecules necessary for the origins of life was demonstrated in paste conditions after a wet-to-dry transition. Integration of this chemistry into a wet/dry cycle by redissolving the paste in a cyanate solution recycled the remaining orthophosphate back into an activated form via the regeneration of imidazole phosphate. Repeated cycling led to an accretion of phosphorylated compounds and therefore can maximize the use of scarce resources such as orthophosphate. This physicochemical orthophosphate cycle could provide a continuous supply of phosphorylated organic compounds for prebiotic chemistry upon the early Earth.

The simplicity of this system makes it ideal for prebiotic chemistry. Only four simple, prebiotically plausible reagents (orthophosphate, cyanate, imidazole and a nucleophile) are required along with a wet/dry cycle, in order to perform all steps of the physicochemical orthophosphate cycle. Importantly, this approach uses orthophosphate-the most likely source of phosphorus on the early Earth and the most thermodynamically favoured oxidation state of phosphorus in aqueous solution $6,57,58$. Moreover, the activation of orthophosphate and its storage in imidazole phosphate occurs in aqueous solution under mild conditions at $22^{\circ} \mathrm{C}$ and $\mathrm{pH} 7.0-8.0$. In principle, this system is capable of capturing solar energy and storing it within imidazole phosphate as cyanate can be formed via a photocatalysed reaction between hydrogen cyanide and water in the presence of an
$\left[\mathrm{Fe}(\mathrm{CN})_{6}\right]^{3-}$ or $\left[\mathrm{Cu}_{2}(\mathrm{CN})_{6}\right]^{2-}$ catalyst ${ }^{49,59}$. Also, spark discharge experiments (simulating lightning) with $\mathrm{N}_{2}, \mathrm{CO}_{2}$ and $\mathrm{H}_{2}$ have demonstrated the formation of cyanate in up to $12 \mathrm{mM}$ concentrations, which is in close alignment with the cyanate concentrations of $20 \mathrm{mM}$ used in Fig. 3l, $\mathrm{m}^{60}$. The utilization of a wet/dry cycle is a well-established means by which to drive a range of prebiotically plausible reaction processes and allows this physicochemical orthophosphate cycle to function at much lower reagent concentrations by taking advantage of how solutes concentrate as solvent evaporates $47,48,61,62$.

To conclude, we have demonstrated a key strategy for how to construct prebiotically plausible chemical systems that capture chemical energy used for the activation of phosphate, store it within a KSTA molecule, which can accumulate, and then later channel that energy into the phosphorylation of the building blocks of life during a wet/dry cycle. More broadly, our approach to constructing the system harnesses the interplay between the kinetic and thermodynamic aspects of chemical reactions and the environmental processes which alter these factors. Considering the prominent role of other KSTA molecules in extant life, such as acetyl CoA, SAM and $\mathrm{NAD}(\mathrm{P}) \mathrm{H}$, we believe that our strategy can be used to assemble other KSTA-based prebiotic systems with functions beyond phosphorylation. The KSTA molecules in extant life play an essential role in keeping a cell in an out-ofequilibrium state - a characteristic feature of living systems. Our demonstration that this KSTA strategy would be viable on the early Earth therefore shows how a prebiotic system could access and then persist in an out-of-equilibrium state, akin to life.

\section{Methods}

Formation of imidazole phosphate from cyanate, orthophosphate and imidazole in aqueous solution. A $450 \mathrm{mM}$ potassium cyanate, $150 \mathrm{mM}$ sodium phosphate and $1.0 \mathrm{M}$ imidazole solution was prepared by dissolving $36.5 \mathrm{mg}$ ( $0.45 \mathrm{mmol}, 3$ eq. $)$ potassium cyanate and $26.7 \mathrm{mg}(0.15 \mathrm{mmol}, 1 \mathrm{eq}$.$) sodium$ phosphate monobasic anhydrous in $1.0 \mathrm{~mL}$ of $1.0 \mathrm{M}$ imidazole buffer $(1.00 \mathrm{mmol}$, 6.67 eq.) containing $50 \mathrm{mM}$ HMPA (hexamethylphosphoramide) internal standard at $\mathrm{pH} 6.17$ in 9:1 $\mathrm{H}_{2} \mathrm{O}: \mathrm{D}_{2} \mathrm{O}$. The $\mathrm{pH}$ of the solution at the start was $\mathrm{pH} 6.37$. The reaction was followed by ${ }^{31} \mathrm{P}-\mathrm{NMR}$ and ${ }^{1} \mathrm{H}-\mathrm{NMR}$ spectroscopy, measuring spectra at a series of time points over the course of 16 days. The $\mathrm{pH}$ of the sample was measured at time points over the course of the reaction. An identical procedure was followed for the reactions with starting pHs of 6.52, 6.73, 7.00 and 7.32. See SI Section S2.

Phosphorylation of prebiotically important organic compounds after a wet-todry transition. Here, $24.2 \mathrm{mg}(0.13 \mathrm{mmol}, 1 \mathrm{eq}$. $)$ of calcium imidazole phosphate (see SI Section S3 for the synthesis of calcium imidazole phosphate) and $98.5 \mathrm{mg}$ $(0.65 \mathrm{mmol}, 5 \mathrm{eq}$.) of D-ribose were dissolved in $2.0 \mathrm{~mL}$ of MilliQ water to give a $65 \mathrm{mM}$ calcium imidazole phosphate and a $325 \mathrm{mM}$ D-ribose solution. The solution was added to a petri dish and left with the lid off to dry at $22^{\circ} \mathrm{C}$ for $24 \mathrm{~h}$. The resulting paste was scraped from the petri dish and placed into an Eppendorf. The Eppendorf was spun on a centrifuge for $5 \mathrm{~min}$ at $5590 \times \mathrm{g}$ in order to move all paste to the bottom of the Eppendorf and compact it together in order to remove air pockets introduced during the transfer of the paste to the Eppendorf. An 10 mg sample of the paste was taken to record the extent of phosphorylation after the wetto-dry transition to a paste. The Eppendorf was placed in a thermoshaker at $50^{\circ} \mathrm{C}$ and 600 r.p.m. for up to $168 \mathrm{~h}$. The reaction was followed by periodically removing samples $(\sim 10 \mathrm{mg})$, dissolving them in $0.5 \mathrm{~mL}$ of $0.5 \mathrm{M}$ citric acid buffer at $\mathrm{pH} 6.85$ and 9:1 $\mathrm{H}_{2} \mathrm{O}: \mathrm{D}_{2} \mathrm{O}$, and analysing them with ${ }^{31} \mathrm{P}-\mathrm{NMR}$ and ${ }^{1} \mathrm{H}-\mathrm{NMR}$, and ${ }^{1} \mathrm{H}^{31} \mathrm{P}$ HMBC (Heteronuclear Multiple Bond Correlation) NMR spectroscopy. The citric acid buffer chelated calcium ions enabling full solubilization of all calcium phosphate salts. Reactions with other nucleophiles were conducted using an identical procedure unless otherwise specified in the SI Section S7.

Physicochemical orthophosphate cycle with three transverses of the cycle. A $450 \mathrm{mM}$ potassium cyanate and a $150 \mathrm{mM}$ sodium phosphate dibasic solution was prepared by dissolving $730.1 \mathrm{mg}(9.0 \mathrm{mmol}, 3 \mathrm{eq}$.$) of potassium cyanate and$ $534.0 \mathrm{mg}$ ( $3.0 \mathrm{mmol}, 1$ eq.) of sodium phosphate dibasic dihydrate salt in $20.0 \mathrm{~mL}$ of $1.0 \mathrm{M}$ imidazole solution (20 mmol, 6.67 eq.) in 9:1 $\mathrm{H}_{2} \mathrm{O}: \mathrm{D}_{2} \mathrm{O}$. Then, $276.3 \mathrm{mg}$ ( $3.0 \mathrm{mmol}$ ) of glycerol was dissolved in $4.0 \mathrm{~mL}$ of this $450 \mathrm{mM}$ potassium cyanate, $150 \mathrm{mM}$ sodium phosphate dibasic and $1.0 \mathrm{M}$ imidazole solution. The $\mathrm{pH}$ of the solution was measured and was typically in the region of $\mathrm{pH} 8.5-10.0$. The $\mathrm{pH}$ of the solution was adjusted to $\mathrm{pH} 7.3$ using $5 \mathrm{M}$ hydrochloric acid and $5 \mathrm{M}$ sodium hydroxide solutions. The solution was left to react for $24 \mathrm{~h}$, in order to accumulate 
imidazole phosphate, and $0.5 \mathrm{~mL}$ of the solution was placed in an NMR tube and the reaction was followed by ${ }^{31} \mathrm{P}-\mathrm{NMR}$ and ${ }^{1} \mathrm{H}$-NMR spectroscopy.

After $24 \mathrm{~h}$, the solution in the NMR tube was recombined with the rest of the solution and the solution was placed into a petri dish and left with the lid off to dry at $22{ }^{\circ} \mathrm{C}$ for $24 \mathrm{~h}$. The resulting paste was scraped from the petri dish and placed into an Eppendorf (an NMR sample of the paste was also taken). The Eppendorf was spun on a centrifuge for $3 \mathrm{~min}$ at $5590 \times g$ in order to move all the paste to the bottom of the Eppendorf and compact it together in order to remove air pockets introduced during the transfer of the paste to the Eppendorf. The Eppendorf was placed on a thermoshaker at $50^{\circ} \mathrm{C}$ and 600 r.p.m. for $24 \mathrm{~h}$. The reaction was followed by periodically removing samples $(\sim 10 \mathrm{mg})$, dissolving them in $0.5 \mathrm{~mL}$ of $0.5 \mathrm{M}$ citric acid buffer at $\mathrm{pH} 6.85$ and $9: 1 \mathrm{H}_{2} \mathrm{O}: \mathrm{D}_{2} \mathrm{O}$ and analysing them with ${ }^{31} \mathrm{P}-\mathrm{NMR}$ and ${ }^{1} \mathrm{H}-\mathrm{NMR}$, and ${ }^{1} \mathrm{H}{ }^{31} \mathrm{P}$ HMBC NMR spectroscopy. After $24 \mathrm{~h}$, the reaction was removed from the thermoshaker and allowed to cool to room temperature.

To complete the first cycle and start the second cycle, the paste was redissolved in $4.0 \mathrm{~mL}$ of $450 \mathrm{mM}$ potassium cyanate solution (prepared from $730.1 \mathrm{mg}$ $\left(9.0 \mathrm{mmol}\right.$ ) of potassium cyanate in $20.0 \mathrm{~mL}$ of $\left.9: 1 \mathrm{H}_{2} \mathrm{O}: \mathrm{D}_{2} \mathrm{O}\right)$. The $\mathrm{pH}$ of the solution was adjusted to $\mathrm{pH}$ 7.3. For the second cycle, the same steps of leaving the solution to react for $24 \mathrm{~h}$, drying to a paste for $24 \mathrm{~h}$ and heating to $50^{\circ} \mathrm{C}$ for $24 \mathrm{~h}$, as detailed above, were followed. After the second cycle was completed, a third cycle was performed using an identical procedure. Reactions with other nucleophiles were conducted using an identical procedure unless otherwise specified in the Supporting Information. For physicochemical orthophosphate cycles performed with different concentrations of reagents (in Fig. 3), similar procedures were used (see SI Sections S7.11 and S7.12 for details).

Further details of all experimental methods are included in the Supporting Information.

\section{Data availability}

The authors declare that all data supporting the findings of this study are available within the paper and Supplementary Information.

Received: 17 May 2021; Accepted: 18 August 2021;

Published online: 17 September 2021

\section{References}

1. Westheimer, F. H. Why nature chose phosphates. Science 235, 1173 (1987)

2. Kamerlin, S. C. L., Sharma, P. K., Prasad, R. B. \& Warshel, A. Why nature really chose phosphate. Q. Rev. Biophys. 46, 1-132 (2013).

3. Pasek, M. A. Thermodynamics of prebiotic phosphorylation. Chem. Rev. 120 , 4690-4706 (2020).

4. Orgel, L. E. Evolution of the genetic apparatus. J. Mol. Biol. 38, 381-393 (1968).

5. Liu, Z., Rossi, J.-C. \& Pascal, R. How prebiotic chemistry and early life chose phosphate. Life 9, 26 (2019).

6. Pasek, M. A., Gull, M. \& Herschy, B. Phosphorylation on the early earth. Chem. Geol. 475, 149-170 (2017).

7. Lohrmann, R. \& Orgel, L. E. Prebiotic activation processes. Nature 244, 418-420 (1973).

8. Burcar, B. et al. Darwin's warm little pond: a one-pot reaction for prebiotic phosphorylation and the mobilization of phosphate from minerals in a ureabased solvent. Angew. Chem. Int. Ed. 55, 13249-13253 (2016).

9. Burcar, B. et al. A stark contrast to modern Earth: phosphate mineral transformation and nucleoside phosphorylation in an iron- and cyanide-rich early Earth scenario. Angew. Chem. Int. Ed. 58, 16981-16987 (2019).

10. Powner, M. W., Gerland, B. \& Sutherland, J. D. Synthesis of activated pyrimidine ribonucleotides in prebiotically plausible conditions. Nature 459, 239-242 (2009).

11. Kim, H.-J. et al. Evaporite borate-containing mineral ensembles make phosphate available and regiospecifically phosphorylate ribonucleosides: borate as a multifaceted problem solver in prebiotic chemistry. Angew. Chem. Int. Ed. 55, 15816-15820 (2016).

12. Maheen, G. et al. Mimicking the prebiotic acidic hydrothermal environment: one-pot prebiotic hydrothermal synthesis of glucose phosphates. Heteroat. Chem. 22, 186-191 (2011)

13. Fiore, M. et al. Giant vesicles from rehydrated crude mixtures containing unexpected mixtures of amphiphiles formed under plausibly prebiotic conditions. Org. Biomol. Chem. 15, 5096-5096 (2017).

14. Reimann, R. \& Zubay, G. Nucleoside phosphorylation: a feasible step in the prebiotic pathway to RNA. Orig. Life Evol. Biosph. 29, 229-247 (1999).

15. Gull, M., Zhou, M., Fernández, F. M. \& Pasek, M. A. Prebiotic phosphate ester syntheses in a deep eutectic solvent. J. Mol. Evol. 78, 109-117 (2014)
16. Gull, M., Cafferty, J. B., Hud, V. N. \& Pasek, A. M. Silicate-promoted phosphorylation of glycerol in non-aqueous solvents: a prebiotically plausible route to organophosphates. Life 7, 29 (2017).

17. Karki, M., Gibard, C., Bhowmik, S. \& Krishnamurthy, R. Nitrogenous derivatives of phosphorus and the origins of life: plausible prebiotic phosphorylating agents in water. Life 7, 32 (2017).

18. Krishnamurthy, R., Guntha, S. \& Eschenmoser, A. Regioselective aphosphorylation of aldoses in aqueous solution. Angew. Chem. Int. Ed. 39, 2281-2285 (2000)

19. Coggins, A. J. \& Powner, M. W. Prebiotic synthesis of phosphoenol pyruvate by a-phosphorylation-controlled triose glycolysis. Nat. Chem. 9, 310-317 (2017).

20. Gibard, C., Bhowmik, S., Karki, M., Kim, E.-K. \& Krishnamurthy, R. Phosphorylation, oligomerization and self-assembly in water under potential prebiotic conditions. Nat. Chem. 10, 212-217 (2018).

21. Gibard, C. et al. Geochemical sources and availability of amidophosphates on the early Earth. Angew. Chem. Int. Ed. 58, 8151-8155 (2019).

22. Ashe, K. et al. Selective prebiotic synthesis of phosphoroaminonitriles and aminothioamides in neutral water. Commun. Chem. 2, 23 (2019).

23. Jiménez, E. I., Gibard, C. \& Krishnamurthy, R. Prebiotic phosphorylation and concomitant oligomerization of deoxynucleosides to form DNA. Angew. Chem. Int. Ed. 60, 10775-10783 (2021).

24. Kee, T. P. et al. Phosphate activation via reduced oxidation state phosphorus (P). Mild routes to condensed-P energy currency molecules. Life 3, 386-402 (2013).

25. Britvin, S. N. et al. Cyclophosphates, a new class of native phosphorus compounds, and some insights into prebiotic phosphorylation on early Earth. Geology 49, 382-386 (2020).

26. Walsh, C. T., Tu, B. P. \& Tang, Y. Eight kinetically stable but thermodynamically activated molecules that power cell metabolism. Chem. Rev. 118, 1460-1494 (2018).

27. Lipmann, F. in Advances in Enzymology and Related Areas of Molecular Biology (eds Nord, F. F. \& Werkman, C. H.) Vol. 1, 99-162 (Wiley, 1941).

28. Pascal, R. Kinetic barriers and the self-organization of life. Isr. J. Chem. 55, 865-874 (2015)

29. Danger, G., d'Hendecourt, L. L. S. \& Pascal, R. On the conditions for mimicking natural selection in chemical systems. Nat. Rev. Chem. 4, 102-109 (2020).

30. Sutherland, J. D. Opinion: studies on the origin of life - the end of the beginning. Nat. Rev. Chem. 1, 0012 (2017).

31. Islam, S. \& Powner, M. W. Prebiotic systems chemistry: complexity overcoming clutter. Chem 2, 470-501 (2017).

32. Jones, M. E. \& Lipmann, F. Chemical and enzymatic synthesis of carbamyl phosphate. Proc. Natl Acad. Sci. USA 46, 1194 (1960).

33. Allen, C. M. \& Jones, M. E. Decomposition of carbamylphosphate in aqueous solutions. Biochemistry 3, 1238-1247 (1964).

34. Jencks, W. P. \& Gilchrist, M. Reactions of nucleophilic reagents with phosphoramidate 1. J. Am. Chem. Soc. 87, 3199-3209 (1965).

35. Cooperman, B. S. \& Lloyd, G. J. Nucleophilic attack by zinc(II)-pyridine-2carbaldoxime anion on phosphorylimidazole. Model for enzymic phosphate transfer. J. Am. Chem. Soc. 93, 4883-4889 (1971).

36. Admiraal, S. J. \& Herschlag, D. Catalysis of phosphoryl transfer from ATP by amine nucleophiles. J. Am. Chem. Soc. 121, 5837-5845 (1999).

37. Jencks, W. P. in Handbook of Biochemistry and Molecular Biology Vol. 1, 296-304 (CRC, 1976).

38. Oró, J., Basile, B., Cortes, S., Shen, C. \& Yamrom, T. The prebiotic synthesis and catalytic role of imidazoles and other condensing agents. Orig. Life Evol. Biosph. 14, 237-242 (1984).

39. Kobayashi, K., Tsuchiya, M., Oshima, T. \& Yanagawa, H. Abiotic synthesis of amino acids and imidazole by proton irradiation of simulated primitive earth atmospheres. Orig. Life Evol. Biosph. 20, 99-109 (1990).

40. Rosenberg, T. A simple preparation method for diphosphoimidazole. Arch. Biochem. Biophys. 105, 315-318 (1964).

41. Bonfio, C. et al. Length-selective synthesis of acylglycerol-phosphates through energy-dissipative cycling. J. Am. Chem. Soc. 141, 3934-3939 (2019).

42. Griesser, H. et al. Ribonucleotides and RNA promote peptide chain growth. Angew. Chem. Int. Ed. 56, 1219-1223 (2017).

43. Canavelli, P., Islam, S. \& Powner, M. W. Peptide ligation by chemoselective aminonitrile coupling in water. Nature 571, 546-549 (2019)

44. Foden, C. S. et al. Prebiotic synthesis of cysteine peptides that catalyze peptide ligation in neutral water. Science 370, 865 (2020).

45. Muchowska, K. B., Varma, S. J. \& Moran, J. Synthesis and breakdown of universal metabolic precursors promoted by iron. Nature 569, 104-107 (2019).

46. Stubbs, R. T., Yadav, M., Krishnamurthy, R. \& Springsteen, G. A plausible metal-free ancestral analogue of the Krebs cycle composed entirely of aketoacids. Nat. Chem. 12, 1016-1022 (2020).

47. Becker, S. et al. Wet-dry cycles enable the parallel origin of canonical and noncanonical nucleosides by continuous synthesis. Nat. Commun. 9, 163 (2018). 
48. Becker, S. et al. Unified prebiotically plausible synthesis of pyrimidine and purine RNA ribonucleotides. Science 366, 76 (2019).

49. Ritson, D. \& Sutherland, J. D. Prebiotic synthesis of simple sugars by photoredox systems chemistry. Nat. Chem. 4, 895-899 (2012).

50. Schwartz, A. W. Prebiotic phosphorylation-nucleotide synthesis with apatite. Biochim. Biophys. Acta 281, 477-480 (1972).

51. Weber, A. L. Formation of pyrophosphate, tripolyphosphate, and phosphorylimidazole with the thioester, N, S-diacetylcysteamine, as the condensing agent. J. Mol. Evol. 18, 24-29 (1981).

52. Lohrmann, R. \& Orgel, L. E. Prebiotic synthesis: phosphorylation in aqueous solution. Science 161, 64 (1968).

53. Keefe, A. D. \& Miller, S. L. Are polyphosphates or phosphate esters prebiotic reagents? J. Mol. Evol. 41, 693-702 (1995).

54. Yamagata, Y. Prebiotic formation of ADP and ATP from AMP, calcium phosphates and cyanate in aqueous solution. Orig. Life Evol. Biosph. 29, 511-520 (1999).

55. Hagan, W. J., Parker, A., Steuerwald, A. \& Hathaway, M. Phosphate solubility and the cyanate-mediated synthesis of pyrophosphate. Orig. Life Evol. Biosph. 37, 113-122 (2007).

56. Liu, Z. et al. Harnessing chemical energy for the activation and joining of prebiotic building blocks. Nat. Chem. 12, 1023-1028 (2020).

57. Ritson, D. J., Mojzsis, S. J. \& Sutherland, J. D. Supply of phosphate to early Earth by photogeochemistry after meteoritic weathering. Nat. Geosci. 13, 344-348 (2020).

58. Toner, J. D. \& Catling, D. C. A carbonate-rich lake solution to the phosphate problem of the origin of life. Proc. Natl Acad. Sci. USA 117, 883 (2020).

59. Saygin, Ö. Nonenzymatic photophosphorylation with visible light. Naturwissenschaften 68, 617-619 (1981).

60. Yamagata, Y. \& Mohri, T. Formation of cyanate and carbamyl phosphate by electric discharges of model primitive gas. Orig. Life Evol. Biosph. 12, 41-44 (1982).

61. Forsythe, J. G. et al. Ester-mediated amide bond formation driven by wet-dry cycles: a possible path to polypeptides on the prebiotic Earth. Angew. Chem. Int. Ed. 54, 9871-9875 (2015).

62. Campbell, T. D. et al. Prebiotic condensation through wet-dry cycling regulated by deliquescence. Nat. Commun. 10, 4508 (2019).

\section{Acknowledgements}

We thank Dr. Paul White for all his help with the NMR studies. This work was supported by funding from the Simons Collaboration on the Origins of Life (SCOL [award 477123] to W.T.S.H.).

\section{Author contributions}

O.R.M. and W.T.S.H. conceived the project. O.R.M. and I.B.A.S. designed and performed experiments and data analysis. O.R.M., W.T.S.H. and I.B.A.S. wrote the manuscript. W.T.S.H. supervised the study.

\section{Competing interests}

The authors declare no competing interests.

\section{Additional information}

Supplementary information The online version contains supplementary material available at https://doi.org/10.1038/s41467-021-25555-x.

Correspondence and requests for materials should be addressed to Wilhelm T. S. Huck.

Peer review information Nature Communications thanks the anonymous reviewer(s) for their contribution to the peer review of this work.

Reprints and permission information is available at http://www.nature.com/reprints

Publisher's note Springer Nature remains neutral with regard to jurisdictional claims in published maps and institutional affiliations.

\begin{abstract}
(c) (i) Open Access This article is licensed under a Creative Commons Attribution 4.0 International License, which permits use, sharing, adaptation, distribution and reproduction in any medium or format, as long as you give appropriate credit to the original author(s) and the source, provide a link to the Creative Commons license, and indicate if changes were made. The images or other third party material in this article are included in the article's Creative Commons license, unless indicated otherwise in a credit line to the material. If material is not included in the article's Creative Commons license and your intended use is not permitted by statutory regulation or exceeds the permitted use, you will need to obtain permission directly from the copyright holder. To view a copy of this license, visit http://creativecommons.org/ licenses/by/4.0/.
\end{abstract}

(C) The Author(s) 2021 